\title{
A Central Apnoea Extension Alarm
}

\author{
Mr G Brown
}

Dr C Rajagopal, MBBS, MRCGP, DCCH

Col D Russell, MBBS, DLO, RAMC

Duchess of Kent's Military Hospital, Catterick Garrison

\begin{abstract}
A central apnoea alarm designed to monitor the breathing of infants placed in four separate cubicles is described. It has been found to be effective and inexpensive, costing about $£ 50$.
\end{abstract}

\section{Introduction}

The need for a central monitor was felt during a winter period when infants with whooping cough required monitoring for apnoea. The prime advantage of such a device is that the nursing staff, especially at night, can keep a vigil without having to stay close to the cubicles. The loud alarm should be heard at the end of an ordinary 20 bedded children's ward.

The Central Apnoea Extension Alarm (CAEA) consists of a power on/off switch, light emitting diode (LED), test/reset switch and audible warning device. The design incorporates four channels each with a memory LED (yellow) and a tricolour LED (green, red and yellow). The CAEA is positioned on the wall facing the Paediatric Nurses Station. The Vickers Mk III apnoea alarm placed in the cubicle of the infant is connected to a wall plug. Cables from each cubicle feed signals into the corresponding channel of the CAEA. The tricolour LED flashes green for every breathing movement of the infant. Apnoea is shown as a continuous red illuminating memory LED and sounding the audible alarm.

The CAEA has been found to be of use in infants who do not need intensive nursing but require monitoring of apnoea in those recovering from respiratory distress syndrome. In a Special Care Baby Unit nursery, premature infants no longer requiring intensive support could be monitored for apnoea. In the past six months, during the trial period, we have monitored prematurely born infants prior to discharge, infants with near miss cot death and those in the post operative period. It has been found to be valuable and we have not come across any defects.

It is possible to extend this monitoring to more babies by incorporating further channels. The CAEA - designed and built by GB - is under consideration by the Army Departmental Technical Suggestions and Awards Scheme.

\section{Acknowledgements}

We thank Miss $\mathrm{J}$ Bell for secretarial assistance.

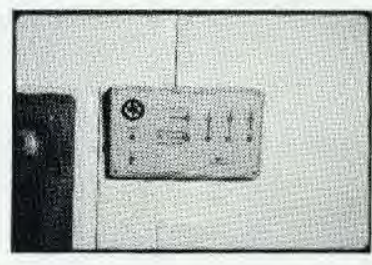

Central apnoea extension alarm located in the Nursing Station.

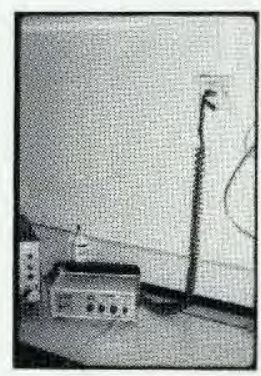

Vickers Mk III Apnoea Alarm connected to the cable. 